\title{
Bulk phantom fields, increasing warp factors and fermion localisation
}

\author{
Ratna Koley * and Sayan Kar ${ }^{\dagger}$ \\ Department of Physics and Centre for Theoretical Studies \\ Indian Institute of Technology, Kharagpur 721 302, India
}

\begin{abstract}
A bulk phantom scalar field (with negative kinetic energy) in a sine-Gordon type potential is used to generate an exact thick brane solution with an increasing warp factor. It is shown that the growing nature of the warp factor allows the localisation of massive as well as massless spinhalf fermions on the brane even without any additional non-gravitational interactions. The exact solutions for the localised massive fermionic modes are presented and discussed. The inclusion of a fermion-scalar Yukawa coupling appears to change the mass spectrum and wave functions of the localised fermion though it does not play the crucial role it did in the case of a decreasing warp factor.
\end{abstract}

\footnotetext{
* Electronic address: ratna@cts.iitkgp.ernet.in

$\dagger$ †lectronic address : sayan@cts.iitkgp.ernet.in
} 
An important issue in the study of braneworld models [1] is the question of localization of the Standard Model fields on the brane. This problem was addressed, many years ago, by Rubakov and Shaposhnikov and independently by Akama [2] though the effect of higher dimensional gravity was not included there. With the advent of Randall-Sundrum (RS) models where a warped higher dimensional background (bulk) geometry is assumed, there has been renewed interest along these directions. The extra dimension in the RS scenario can be finite (RS-I) or infinite (RS-II). We shall mainly deal with RS-II type models in this article.

Our first goal here is to generate a thick brane realisation of RS-II with a growing warp factor. Such increasing warp factor solutions have been obtained by several authors [3]. Here we propose a hypothetical scalar field with a wrong-sign (negative) kinetic energy in the bulk. Such strange 'matter' fields have been named as the 'phantom' or 'ghost' and has received increasing attention among theorists because of their potential in explaining the observational accelerated expansion of our universe. It has been introduced by Caldwell [4] and others [5] in the context of four dimensional cosmology. The phantom field obviously violates all versions of the energy conditions-strong, weak, null as well as dominant. Given our limitation in obtaining any experimental evidence for bulk matter one may propose a model in which the bulk consists of a energy condition violating scalar field. If the four dimensional consequences of such exoticity in the fifth seem reasonable, we should perhaps not worry too much about what is really there in the fifth dimension. Having found the warp factor, we then look at the localisation of fermions. It turns out that the fermion spectrum can be exactly obtained and there do exist localised states. Furthermore, we show that massless as well as massive modes of the spin 1/2 fermion can be confined on the 3-brane even without any kind of Yukawa interaction between the bulk and brane matter fields.

Let us begin with the action for the bulk spacetime which consists of a negative cosmological constant, gravity and a bulk scalar field with a sine-Gordon potential:

$$
S=\int\left[\frac{1}{2 \kappa_{5}^{2}}(R-2 \Lambda)+\frac{1}{2}(\nabla \phi)^{2}-V(\phi)\right] \sqrt{-g} d^{5} x
$$

Note that the kinetic energy of the bulk scalar field is set to be negative.

Our setup has a single brane embedded in the bulk. The ansatze for a warped spacetime following standard terminology, is given by: 


$$
d s^{2}=d \sigma^{2}+e^{-2 f(\sigma)} \eta_{i j} d x^{i} d x^{j}
$$

where, the signature convention is $(-++++)$ and the small Latin indices $i, j=0,1,2,3$ refer to the brane coordinates. The scalar field will be assumed to be a function of the extra coordinates only.

In this framework, the Einstein scalar system reduces to the following set of coupled, nonlinear ordinary differential equations :

$$
\begin{aligned}
f^{\prime \prime} & =-a \phi^{2} \\
f^{\prime 2} & =\frac{a}{4}\left(-\phi^{2}-2 V\right)-\frac{\Lambda}{6} \\
\phi^{\prime \prime}-4 f^{\prime} \phi^{\prime} & =-\frac{d V}{d \phi}
\end{aligned}
$$

where $a=\kappa_{5}^{2} / 3$.

The scalar field (Klein-Gordon) equation follows from the first two Einstein equations and is not independent. This system of equations can be solved exactly for a sine-Gordon potential given by

$$
V(\phi)=B\left(1+\cos \frac{2 \phi}{A}\right)
$$

The explicit forms of the solutions for the scalar field and the warp factor are given as

$$
\begin{array}{r}
f(\sigma)=-\frac{a}{\kappa_{1}} \sqrt{\frac{|\Lambda|}{6}} \ln \cosh \left(\frac{\kappa_{1}}{a} \sigma\right) \\
\phi(\sigma)=2 A \tan ^{-1}\left(\exp \frac{\kappa_{1}}{a} \sigma\right)-\frac{\pi A}{2}
\end{array}
$$

where, $\kappa_{1}=\frac{1}{A^{2}} \sqrt{\frac{\mid \Lambda \Lambda}{6}}$. And the constant $\mathrm{B}$ in the sine-Gordon potential is given as : $B=\frac{|\Lambda|}{6 a^{2}}\left(a-\frac{1}{4 A^{2}}\right)$. Note here that a decreasing warp factor is obtainable through a usual (positive kinetic energy) scalar field in a SG potential. This is discussed by us in 7].

It is important to note the presence of a negative cosmological constant in both the warp factor and the soliton. This model with a bulk SG potential provides a 'thick brane' scenario where the SG field and its soliton configuration dynamically generate this domain wall configuration in the background warped geometry. In addition as is obvious from the 
functional from of $f$, there is no discontinuity in the derivative of $f$ at the location of the brane. The warp factor is smooth everywhere and has all the necessary features.

The line element in the warped bulk space time is given by

$$
d s^{2}=d \sigma^{2}+\cosh ^{2 \nu}\left(\frac{\kappa_{1}}{a} \sigma\right) \eta_{i j} d x^{i} d x^{j}
$$

where $\nu=\frac{a}{\kappa_{1}} \sqrt{\frac{|\Lambda|}{6}}=A^{2} a$. The distinct feature of this metric is that the warp factor is a growing function of the extra coordinate. The phantom field is responsible for such a line element for the background geometry. As we show later, the localisation of spinor fields on the brane without any non-gravitational interaction with the bulk scalar is achieved largely due to the growing nature of the warp factor.

Notice that the metric is completely non-singular for the full domain of the fifth coordinate. It describes a space of negative Ricci curvature where $R$ is given by :

$$
R=-4 \frac{|\Lambda|}{6}\left[\left(5-\frac{2}{\nu}\right) \tanh ^{2}\left(\frac{\kappa_{1}}{a} \sigma\right)+\frac{2}{\nu}\right]
$$

It is straightforward to check that $\mathrm{R}$ is always negative and asymptotically $(\sigma \rightarrow \pm \infty$ ) takes on the value of $-\frac{10}{3}|\Lambda|$. One can also get an AdS space of constant negative Ricci curvature for the value of the parameter $\nu=2 / 5$.

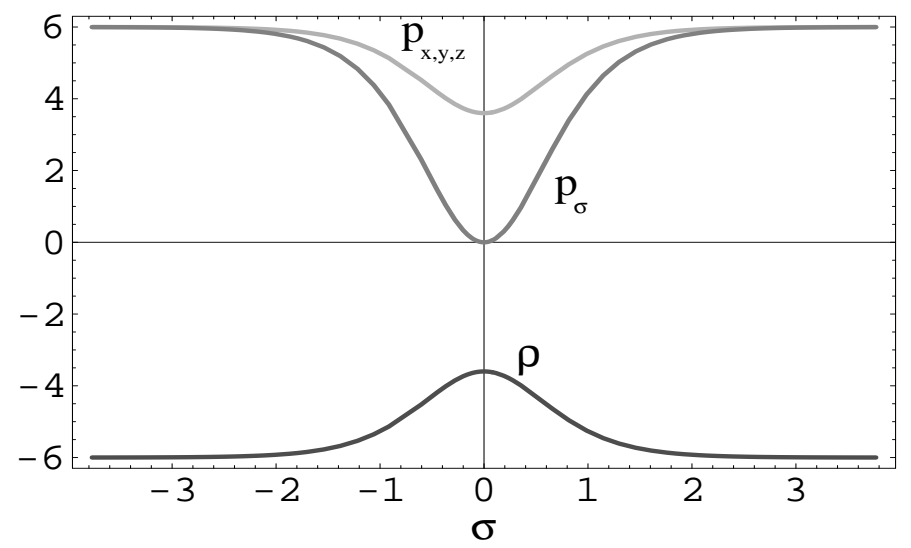

FIG. 1: Variation of the energy density $\rho$ and pressure ( $p_{i}$ and $\left.p_{\sigma}\right)$ with $\sigma$ corresponding to the warped geometry given by the metric in eqn. (9) for $\frac{\kappa_{1}}{a}=\frac{4}{5}$ and $|\Lambda|=6$.

The energy density and pressures, which generate such a line element, are found to be :

$$
\rho=-p_{x, y, z}=-3 \frac{\kappa_{1}}{a} \sqrt{\frac{|\Lambda|}{6}} \operatorname{sech}^{2} \frac{\kappa_{1}}{a} \sigma-|\Lambda| \tanh ^{2} \frac{\kappa_{1}}{a} \sigma
$$




$$
p_{\sigma}=|\Lambda| \tanh ^{2} \frac{\kappa_{1}}{a} \sigma
$$

At $\sigma=0$ (i.e. the location of the brane) we have $p_{x, y, z}=-\rho$ and $p_{\sigma}=0-$ an effective cosmological constant on the 3-brane. The energy density and pressures are plotted in Figure (1). It is worth noting that the matter stress-energy which acts as a source for the warped geometry does not satisfy the Null Energy Condition (NEC $\rightarrow \rho+p_{i} \geq 0$ ). The other energy conditions (such as the Weak Energy Condition and the Dominant Energy Condition) are also viotated. [8].

Let us now focus attention on the localization of spinor fields on the brane. It is known that the kink solutions of the scalar field as given in may trap fermionic zero modes [9]. The bulk fermion coupled to the scalar field in 5D gives rise to two chiral fermionic zero modes in the four dimensions. Depending on the sign of the coupling constant one of these two modes is found to be localized on the brane while the other is delocalized and not normalizable [11]. In all the models discussed till date, the Yukawa coupling with the bulk scalar is capable of localising only a single chiral state (right or left) while fermions of both chiralities would be expected. We will show that in the geometry with an increasing warp factor both the left and right chiral zero modes are confined to the brane even without any coupling with the bulk field. Localised massive modes are also achieved in this background geometry.

The Lagrangian for a Dirac fermion propagating in the five dimensional warped space with the metric (9) is :

$$
\sqrt{-g} \mathcal{L}_{\text {Dirac }}=\sqrt{-g}\left(i \bar{\Psi} \Gamma^{a} \mathcal{D}_{a} \Psi-\eta_{F} \bar{\Psi} \mathrm{F}(\Phi) \Psi\right)
$$

where $g=\operatorname{det}\left(g_{a b}\right)$, is the determinant of full five dimensional metric and $\Phi=\phi / A$. The matrices $\Gamma^{a}=\left(e^{f(\sigma)} \gamma^{\mu},-i \gamma^{5}\right)$ provide a four dimensional representation of the Dirac matrices in five dimensional curved space. Where $\gamma^{\mu}$ and $\gamma^{5}$ are the usual four dimensional Dirac matrices in chiral representation. We also consider a coupling between the bulk scalar and Dirac field. Later we will show that, it is not necessarilly required for the localization of fermions on the brane. However, we will discuss the effect of the coupling on the confinement of different fermionic modes on the brane.

The covariant derivative in 5D curved space for the metric given in Eqn. (9) [10]:

$$
\mathcal{D}_{\mu}=\left(\partial_{\mu}-\frac{1}{2} f^{\prime}(\sigma) e^{-f(\sigma)} \Gamma_{\mu} \Gamma^{4}\right) ; \quad \mathcal{D}_{4}=\partial_{\sigma}
$$


The Dirac Lagrangian in 5D curved spacetime then reduces to the following form

$$
\sqrt{-g} \mathcal{L}_{\text {Dirac }}=e^{-4 f(\sigma)} \bar{\Psi}\left[i e^{f(\sigma)} \gamma^{\mu} \partial_{\mu}+\gamma^{5}\left(\partial_{4}-2 f^{\prime}(\sigma)\right)-\eta_{F} \mathrm{~F}(\Phi)\right] \Psi
$$

The dimensional reduction from $5 \mathrm{D}$ to $4 \mathrm{D}$ is performed in such a way that the standard four dimensional chiral particle theory is recovered. The five dimensional spinor can be decomposed into four dimensional and fifth dimensional parts: $\Psi\left(x^{\mu}, \sigma\right)=\Psi\left(x^{\mu}\right) \xi(\sigma)$. Since the four dimensional massive fermions require both the left and right chiralities it is convenient to organise the spinors with respect to $\Psi_{L}$ and $\Psi_{R}$ which represent four component spinors living in five dimensions given by $\Psi_{L, R}=\frac{1}{2}\left(1 \mp \gamma_{5}\right) \Psi$. Hence the full 5D spinor can be split up in the following way

$$
\Psi\left(x^{\mu}, \sigma\right)=\left(\Psi_{L}\left(x^{\mu}\right) \xi_{L}(\sigma)+\Psi_{R}\left(x^{\mu}\right) \xi_{R}(\sigma)\right)
$$

where $\xi_{L . R}(\sigma)$ satisfy the following eigenvalue equations

$$
\begin{aligned}
& e^{-f(\sigma)}\left[\partial_{\sigma}-2 f^{\prime}(\sigma)-\eta_{F} \mathrm{~F}(\Phi)\right] \xi_{R}(\sigma)=-m \xi_{L}(\sigma) \\
& e^{-f(\sigma)}\left[\partial_{\sigma}-2 f^{\prime}(\sigma)+\eta_{F} \mathrm{~F}(\Phi)\right] \xi_{L}(\sigma)=m \xi_{R}(\sigma)
\end{aligned}
$$

Here $m$ denotes the mass of the four dimensional fermions. The full $5 \mathrm{D}$ action reduces to the standard four dimensional action for the massive chiral fermions, when integrated over the extra dimension [11], if (a) the above equations are satisfied by the bulk fermions and (b) the following orthonormality conditions are obeyed.

$$
\begin{array}{r}
\int_{-\infty}^{\infty} e^{-3 f(\sigma)} \xi_{L_{m}} \xi_{L_{n}} d \sigma=\int_{-\infty}^{\infty} e^{-3 f(\sigma)} \xi_{R_{m}} \xi_{R_{n}} d \sigma=\delta_{m n} \\
\int_{-\infty}^{\infty} e^{-3 f(\sigma)} \xi_{L_{m}} \xi_{R_{n}} d \sigma=0
\end{array}
$$

The dynamical features of the model can thus be obtained from the solutions of the eigenvalue equations (17) and (18).

Let us first focus on massless (i.e. $m=0$ ) fermions for a kink-fermion Yukawa coupling of the form $F(\Phi)=\sin \Phi$. The choice of $F(\Phi)$ is governed by (i) the requirement of $\sigma \rightarrow-\sigma$ symmetry of the effective potential (see Eqn. (23) below) and (ii) exact integrability of the Schrodinger-like equation. One may also consider $F(\Phi)=\Phi$ or any other odd function of 
$\Phi$ but in such cases the equation for the fermion wave function becomes too complicated to integrate analytically. Equation (17) and (18) reduce to two decoupled equations. The asymptotic solutions are

$$
\begin{aligned}
& \xi_{L}(\sigma) \sim e^{-\left(\eta_{F}+2 \sqrt{\frac{\Lambda \Lambda}{6}}\right)|\sigma|} \\
& \xi_{R}(\sigma) \sim e^{\left(\eta_{F}-2 \sqrt{\frac{\Lambda \Lambda}{6}}\right)|\sigma|}
\end{aligned}
$$

It is clear from the above expressions that both the left and right chiral massless modes are normalizable even for $\eta_{F}=0$, This is in accord with the statements on exponentially rising warp factors in [6]. Obviously, restrictions on nongravitational couplings are required in the RS framework (decreasing warp factor) for the fermions to be confined on the brane [11, 12].

We now turn our attention towards the massive fermions. Using the rescaling $\tilde{\xi}_{L . R}(\sigma)=$ $e^{-\frac{3}{2} f(\sigma)} \xi_{L . R}(\sigma)$ and Eqn. (17) and (18) we obtain a second order decoupled equation for the left chiral fermions whereas the right chiral modes can be completely defined from prior knowledge of the left chiral states.

The equation for $\tilde{\xi}_{L}(\sigma)$ can be recast as a generalised Schrödinger equation by the suitable scaling of the function $\tilde{\xi}_{L} \rightarrow \hat{\xi}_{L}(\sigma) e^{f(\sigma)}$, given as follows

$$
\partial_{\sigma}^{2} \hat{\xi}_{L}(\sigma)+\left[m^{2} e^{2 f(\sigma)}+\frac{f^{\prime \prime}(\sigma)}{2}+\eta_{F} \frac{\mathrm{dF}}{\mathrm{d} \Phi} \Phi^{\prime}(\sigma)-\left(\eta_{F} \mathrm{~F}(\Phi)+\frac{f^{\prime}(\sigma)}{2}\right)^{2}\right] \hat{\xi}_{L}(\sigma)=0
$$

The bulk spacetime is $Z_{2}$ symmetric with respect to the brane at $\sigma=0$. Consider the Yukawa coupling, $\mathrm{F}(\Phi)=\sin \Phi$ mentioned earlier. The effective potential acting on the left chiral fermions then shows a minimum at the location of the brane and asymptotically approaches a constant positive value. This functional form is known as the Pösch Teller potential [13] well In the case at hand, this can be written explicitly as :

$$
V_{e f f}(\sigma)=-\left(\eta_{F}^{2}+m^{2}-\frac{b^{2}}{4}\right) \operatorname{sech}^{2}(b \sigma)
$$

where, $b=\frac{\kappa_{1}}{a}$. The nature of the potential is shown in Fig. (2). The depth of the potential increases with increasing coupling constant. It is evident that the modes will be found to be localised on the brane even for $\eta_{F}=0$. We choose to work with the parameter 


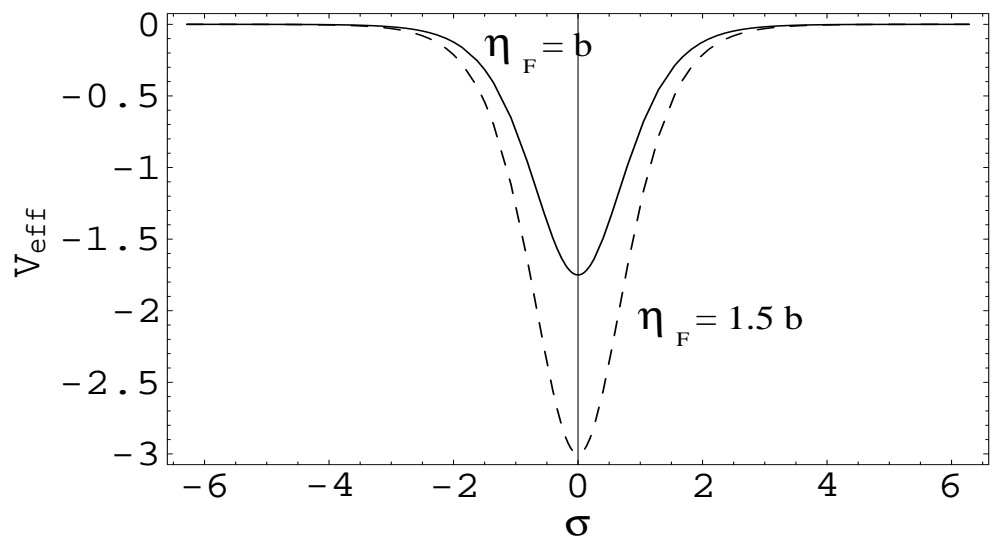

FIG. 2: The effective potential acting on the left chiral fermions plotted as a function of extra dimension $\sigma$ for two different values of coupling constant, $\eta_{F}=\mathrm{b}$ and $1.5 \mathrm{~b}$ which depicts that the depth is greater for stronger coupling. In this case we choose $|\Lambda|=6$ and mass $m=\mathrm{b}$.

$\nu=1$ in our study of the massive modes. This choice is prompted by integrability of the equation, though, with growing warp factors with $\nu \neq 1$ the nature of the potential remains unchanged and our qualitative conclusions will also not be altered.

The Eqn. (23) reduces to the following form

$$
\partial_{\sigma}^{2} \hat{\xi_{L}}(\sigma)+\left(A_{1} \operatorname{sech}^{2}(b \sigma)-A_{2}\right) \hat{\xi_{L}}(\sigma)=0
$$

where, the constants are given by

$$
\begin{aligned}
& A_{1}=\left(m^{2}+\eta_{F}^{2}-\frac{b^{2}}{4}\right) \\
& A_{2}=\left(\eta_{F}-\frac{b}{2}\right)^{2}
\end{aligned}
$$

The exact solutions for the massive modes corresponding to the above equation can be written in terms of the Hypergeometric functions in the following way

$$
\hat{\xi}_{L}(\sigma)=\operatorname{sech}^{\left(\frac{\eta_{F}}{2 b}-\frac{1}{4}\right)}(b \sigma)^{2} F_{1}\left[\epsilon-s, \epsilon+s+1, \epsilon+1, \frac{1}{2}(1-\tanh (b \sigma))\right]
$$

where,

$$
\begin{aligned}
& \epsilon=\left(\frac{\eta_{F}}{b}-\frac{1}{2}\right) \\
& s=\frac{1}{2}\left(-1+\frac{2 \sqrt{m^{2}+\eta_{F}^{2}}}{b}\right)
\end{aligned}
$$




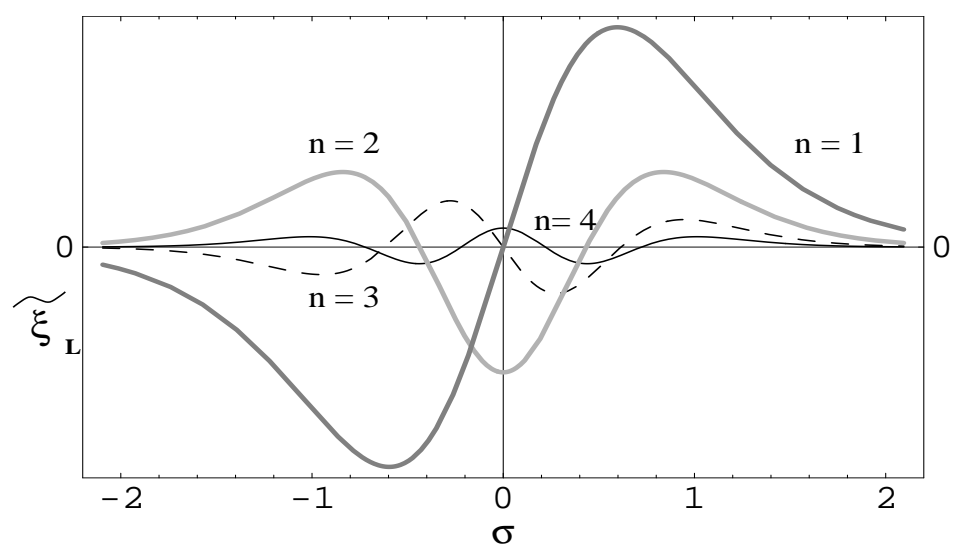

FIG. 3: The wave function corresponding to the massive left chiral fermions, $\tilde{\xi}_{L}$ is plotted as a function of the extra coordinate $\sigma$ for $n=1,2,3$ and 4 and $\eta_{F}=\mathrm{b}, 2 \mathrm{~b}, 3 \mathrm{~b}$ and $4 \mathrm{~b}$ respectively.

The normalization conditions are obviously satisfied by the massive fermions. The modes with mass $\mathrm{m}$ will be found confined to the brane till the well depth is less compared to the kinetic energy of the states. We obtain a mass spectrum from the exact solution obtained in the above case. The parity of the chiral fermions with respect to the transformation $\sigma \rightarrow-\sigma$ can be obtained from the symmetry property of the full five dimensional function $\Psi\left(x^{\mu}, \sigma\right)$. The full function will have a definite parity because of the overall symmetry of the problem under $\sigma \rightarrow-\sigma$. Thus the right and left chiral wavefunctions will be either odd or even. Mass spectrum of the modes can be obtained from condition imposed on the wave function to be well behaved on the brane. The possible values of $m^{2}$ are given by the following expression :

$$
m_{n}^{2}=\left(n^{2}+\frac{2 n \eta_{F}}{b}\right) b^{2}
$$

where $\mathrm{n}=1,2,3,4 \ldots$.

Notice that we have excluded $n=0$. This is because, the second order equation we are solving for is obtained using the restriction $m \neq 0$. For $m=0$, as mentioned before, the first order equations decouple and have to be treated separately. The mass spectrum depends on two parameters $\eta_{F}$ and b (or $|\Lambda|$ ). The earlier models dealing with the localisation of fermions required a large value of coupling constant for the massive modes to be confined around the brane. Here, even for $\eta_{F}=0$, the mass of the fermion is $m_{n} \sim n b$. It is evident from the spectrum in Eqn. (31) that heavier masses can be found on the brane for stronger coupling. 
Let us now summarize pointwise the results obtained in this article and discuss open issues.

(i) An exact thick brane solution with an increasing warp factor is obtained for a bulk scalar field with negative kinetic energy and a sine-Gordon potential.

(ii) Localization of massive and massless fermions are studied in this background geometry. The massless left as well as right chiral modes are found to be normalizable on the brane.

(iii) Massive modes are also confined to the brane. Their mass spectrum is obtained in the presence of a Yukawa coupling though they remain localizable without the coupling.

Our article provides a toy model with a growing warp factor for which we are able to obtain exact analytical results. It is true that growing warp factors might be problematic in other situations such as the hierarchy problem, localisation of gravity. In particular, one may need to consider the two-brane RS1 set-up to avoid embarrasing conclusions related to these problems. In such a set-up one may view the brane on which fermions are localised (via an increasing warp factor) as the 'negative tension' brane with gravity being localised on the 'positive tension' brane situated at a separate location. However, at this stage, when one does not even know clearly whether there is warping in the real world, it is probably useful to keep in mind the full spectrum of possibilities. With this intention, we have, in this article, put forward an exact increasing warp factor solution and discussed one of its consequences. We hope to report more results on similar issues in the near future.

[1] L. Randall and R. Sundrum, Phys. Rev. Lett. 83, 3370 (1999); ibid Phys. Rev. Lett. 83, 4690 (1999) ;

[2] V. A. Rubakov and M. E. Shaposhnikov, Phys. Lett. B 125136 (1983); K. Akama, in Proc. Int. Symp. at Nara, Japan, Springer, (1983) 267

[3] M. Visser, Phys. Letts. B159, 22 (1985) M. Maziashvili.hep-th/0404218; M. Gogberashvili and P. Midodashvili, Phys. Letts. B515, 447 (2001) ibid. Europhys. Letts. 61,208 (2003) ; P. Midodashvili, hep-th/0308051; M. Gogberashvili and D. Singleton, Phys. Rev. D69, 026004 ibid Phys. Letts. B 582, 95 (2004)

[4] R. R. Caldwell, M. Kamionkowski and N. N. Weinberg, astro-ph/0302506 
[5] S. M. Carroll, M Hoffman and M. Trodden, astro-ph/0301273; G. W. Gibbons, hep-th/0302199 H. P. Nilles, Phys. Rep, 110, 1 (1984); M. D. Pollock, Phys. Lett. B 215, 635 (1988); P. Frampton, astro-ph/0209037; V. Sahni and Y. Shtanov, astr-ph/0202346 B. McInnes, JHEP 0208, 029 (2002)

[6] B. Bajc and G. Gababdadze, Phys. Letts. 474, 282 (2000)

[7] R. Koley and S. Kar, Scalar kinks and fermion localisation in warped spacetimes (submitted).

[8] For a useful summary on the details of the energy conditions see Lorentzian Wormholes : from Einstein to Hawking by Matt Visser (AIP, 1995)

[9] R. Jackiw and C. Rebbi, Phys. Rev. D13, 3398 (1976); Y. Grossman and N. Neubert, Phys. Lett. B474 361 (2000)

[10] S. Weinberg, Gravitation and Cosmology (John Wiley and Sons, 1971)

[11] C. Ringeval, P. Peter, J. P. Uzan, Phys. Rev. D 65, 044416 (2002); S. Ichinose, Phys.Rev. D66, 104015 (2002)

[12] S. Randjbar-Daemi and M. Shaposhnikov, Phys. Letts. B492, 361 (2000)

[13] L.D. Landau and E.M. Lifshitz, Quantum Mechanics, Course of Theoretical Physics, Vol. 3, Third Edition (Butterworth - Heinemann) 